\title{
Poisson's equation and generalized axially symmetric potential theory $\left(^{*}\right)$
}

\author{
Nota di R. P. Gillbert (Maryland U.S. A.)
}

\begin{abstract}
In this paper a method is which one may obtain solutions to the non-homogeneous equation of generalized axially given by symmetric potental theory (GASPT). Representation formuloe are obtained and the method is shown to be extendable under certain symmetry conditions to the Poisson equation in three variables.
\end{abstract}

\section{I. - Introduction.}

Professor A. Weinstein [1], [2], [3], [4] has referred to the study of solutions of the partial differential equations

$$
L^{(k)}[\Phi] \equiv \frac{\partial^{2} \Phi}{\partial x^{2}}+\frac{\partial^{2} \Phi}{\partial y^{2}}+\frac{k}{y} \frac{\partial \Phi}{\partial y}=0, \quad k \geq 0
$$

as generalized axially symmetric potential theory (GASPT). This equation is initially arrived at by considering those solutions of the $n$-dimensional LA. PLACE equation which depend solely on

$$
\left.x=x_{1}, \quad y=\left(x_{2}^{2}+\ldots+x_{n}^{2}\right) \quad \text { (in this case } k=n-2\right) .
$$

In previous works this author [5], [6], [7], [8] $\left(^{1}\right)$ has studied properties of the GASPT equation by function theoretic methods based on the use of

(*) This rerearch was supported in part by the United States Air Force through the Air Force Office of Scientific Research of the Air Research and Development Command under grant - AFOSR $62-454$.

(1) For similar investigations of the integral rperator method as applied to GASPT, see Henrici [9], Mackie [10], and Erdélyi [15].

The function-theoretic approach which we use hese is primarilly due to $S$ BERgMar, see for instance [11], [12] and [13]. 
an integral operator $\mathfrak{A}_{k}[f]$, which naps analytic functions $f(\sigma)$ onto solutions of (1)

$$
\begin{gathered}
\Phi(z)=\mathfrak{A}_{k}[f]=\alpha_{k} \int_{+1}^{-1} f(\sigma)\left(\zeta-\zeta^{-1}\right)^{k-1} \frac{d \zeta}{\zeta} \\
\alpha_{k}=\frac{4}{(4 i)^{k} \Gamma\left(\frac{k}{2}\right)^{2}}, \quad \sigma=x+\frac{i y}{2}\left(\zeta+\zeta^{-1}\right), \quad\left|z-z^{0}\right|<\epsilon, \\
z=x+i y, \quad z^{0}=x^{0}+i y^{0}
\end{gathered}
$$

is an initial point of definition for $\Phi(z), \epsilon>0$ is sufficiently small, and integration path is the upper semi-circular arc connecting +1 to -1 .

In this paper we shall investigate the properties of solutions to the non-homogeneous GASPT equation, namely

$$
L^{(k)}[\Phi]=\rho(x, y)
$$

by means of integral operator methods. One notes first, that when $k=0$ equation (3) becomes

$$
\triangle \Phi=P(x, y)
$$

and that by replacing $x, y$ by $x=\frac{z+\bar{z}}{2}, y=\frac{z-\bar{z}}{2 i}$, we may rewrite (4) as

$$
4 \frac{\partial^{2} \Phi}{\partial z \partial \bar{z}}=P(z, \bar{z})
$$

which integrates directly into

$$
\Phi=\int^{\bar{z}} \int^{z} P d z d z+g(z)+\overline{h(z)} .
$$

This method suggests that we consider ihe integral operator $A_{k}[f]$ :

$$
\Phi(z)=A_{k}[f] \equiv \alpha_{k} \int_{+1}^{-1} f\left(\sigma, \sigma^{*}\right)\left(\zeta-\zeta^{-1}\right)^{k-1} \frac{d \zeta}{\zeta}
$$

where $f\left(\sigma, \sigma^{*}\right)$ is an analytic function of the two complex variables $\sigma, \sigma^{*} ; \sigma$ 
is the same as before, $\sigma^{*}=x-\frac{i y}{2}\left(\zeta+\zeta^{-1}\right),\left|z-z^{0}\right|<\epsilon$, and $\epsilon>0$ is sufficiently small ect. It is clear, that if $|\zeta|=1$ and $x, y$ are real then $\sigma^{*}=\bar{\sigma}$. Also, if $\frac{\partial f}{\partial \sigma} \equiv 0$, or $\frac{\partial f}{\partial \sigma^{*}} \equiv 0$, then $t$ is either a function of $\sigma^{*}$ or $\sigma$ respectively; in these cases $A_{k} \rightarrow \mathfrak{A}_{k}$, and $\Phi(2)$ is a solution of (1).

In order to obtain a solution of (3) we apply the operator $L^{(k)}$ to (5) and interchange the orders of integration and differentiation,

$$
\begin{gathered}
L^{(k)}[\Phi]=\alpha_{k} \int_{+1}^{-1} L^{(k)}\left[f\left(\sigma, \sigma^{*}\right)\right]\left(\zeta-\zeta^{-1}\right)^{k-1}= \\
=\frac{\alpha_{k}}{4} \int_{+1}^{-1}\left[-\frac{\partial^{2} f}{\partial \sigma^{2}}\left(\zeta-\zeta^{-1}\right)^{2}+2 \frac{\partial f}{\partial \sigma \partial \sigma^{*}}\left(4+\left[\zeta+\zeta^{-1}\right]^{2}\right)-\right. \\
\left.-\frac{\partial f^{2}}{\partial \sigma^{* 2}}\left(\zeta-\zeta^{-1}\right)^{2}+2 i \frac{k}{y} \frac{\partial f}{\partial \sigma}-2 \frac{i k}{y} \frac{\partial f}{\partial \sigma^{*}}\right]\left(\zeta-\zeta^{-1}\right)^{k-1} \frac{d \zeta}{\zeta}
\end{gathered}
$$

By rearranging terms, and integrating by parts one obtains

$$
\begin{gathered}
L^{(k)}[\Phi]=-\frac{\alpha_{k}}{4} \int_{+1}^{-1} f_{\sigma \sigma}\left(\zeta-\zeta^{-1}\right)^{k-1} \frac{d \zeta}{\zeta}+\frac{i \alpha_{k}}{2 y}\left[f_{\sigma}\left(\zeta-\zeta^{-1}\right)^{k}\right]_{-1}^{+1}- \\
-\frac{i \alpha_{k}}{2 y} \int_{+1}^{-1} \frac{d}{d \zeta} f_{\sigma}\left(\zeta-\zeta^{-1}\right)^{k-1} \frac{d \zeta}{\zeta}-\frac{\alpha_{k}}{4} \int_{+1}^{-1} f_{\sigma^{*} \sigma^{*}}\left(\zeta-\zeta^{-1}\right)^{k+1} \frac{d \zeta}{\zeta}- \\
-\frac{i \alpha_{k}}{2 y}\left[f_{\sigma^{*}}\left(\zeta-\zeta^{-1}\right)^{k}\right]_{-1}^{+1}+\frac{i \alpha_{k}}{2 y} \int_{+1}^{-1} \frac{d}{d \zeta} f_{\sigma^{*}\left(\zeta-\zeta^{-1}\right)^{k} d \zeta+} \\
+\alpha_{k} \int_{+1}^{-1} f_{\sigma^{*} \sigma}\left[2+\frac{1}{2}\left(\zeta+\zeta^{-1}\right)^{2}\right]\left(\zeta-\zeta^{-1}\right)^{k-1} \frac{d \zeta}{\zeta}= \\
=4 \alpha_{k} \int_{+1}^{-1} f_{\sigma^{*} \sigma}\left(\zeta-\zeta^{-1}\right)^{k-1} \frac{d \zeta}{\zeta}=\rho(x, y) .
\end{gathered}
$$

We realize from this, that if $f\left(\sigma, \sigma^{*}\right)$ satisfies the integral equation (7), then $\Phi(z)=A_{k}[f]$ is a solution of equation (3). 
II. - Properties of the operator $A_{k}[f]$.

We first prove

Theorem 1. - In a sufficiently small neighborhood, $N\left(z^{0}\right)$, of an initial point of definition $z^{0}$, the representation

$$
\Phi(z)=A_{k}[f] \equiv \alpha_{k} \int_{+1}^{-1} f\left(\sigma, \sigma^{*}\right)\left(\zeta-\zeta^{-1}\right)^{k-1} \frac{d \zeta}{\zeta},
$$

yields the most general, analytic solution to $L^{(k)}[\Phi]=p(x, y)$. providing that $f$ is contained in the class of analytic functions of two variables $\mathscr{F}$, which satisfy the integral equation,

$$
\rho(x, y)=4 \alpha_{k} \int_{+1}^{-1} \frac{\partial^{2} f}{\partial \sigma \partial \sigma^{*}}\left(\zeta-\zeta^{-1}\right)^{k-1} \frac{d \zeta}{\zeta} .
$$

Proof. - It is clear that if $f \varepsilon \bar{y}$, then $\Phi(z)=A_{k}[f]$ is a solution of (3) if $z \varepsilon N\left(z^{0}\right)$, and $N\left(z^{0}\right)$ is sufficiently small. Furthermore, if $F\left(\sigma, \sigma^{*}\right) \varepsilon \mathfrak{F}$, then $F\left(\sigma, \sigma^{*}\right)=f\left(\sigma, \sigma^{*}\right)+g(\sigma)+h\left(\sigma^{*}\right)$, where $g$ and $h$ are arbitrary analitic functions of $\sigma, \sigma^{*}$ respectively. One then has, that

$$
A_{k}\left[F\left(\sigma, \sigma^{*}\right)\right]=A_{k}\left[f\left(\sigma, \sigma^{*}\right)+g(\sigma)+h\left(\sigma^{*}\right)\right]=A_{k}[f]+\mathfrak{Q}_{k}[g]+\mathfrak{Q}_{k}^{*}[h],
$$

where $\mathfrak{A}_{k}^{*}[h] \stackrel{\text { def }}{=} u(\bar{z})$, if $\mathfrak{A}_{k}[h]=u(z)$.

The difference

$$
A_{k}[F]-A_{k}[f]=\mathfrak{O}_{k}[g]+\mathfrak{Q}_{k}^{*}[h]
$$

represents the general analytic (in $x, y$ ) solution of (1) is some neighborhood $N\left(z^{0}\right)$ of $z^{0}$, since either $\mathfrak{d}_{k}[g]$ or $\mathfrak{G}_{k}^{*}[h]$ represent general solutions of the GASPT equation. (See Gilbert [5], [6], [7]). It follows then that $A_{k}[F]$ represents the most general solution to $(3)$ in a sufficiently small neighborhood $N\left(z^{0}\right)$.

The next problem we consider is the representation of solutions of $L^{(k)}[\Phi]=\rho^{\prime}(x, y)$ by $A_{k}[F]$. For instance, if $F\left(\sigma, \sigma^{*}\right)$ has the series expansion

$$
F\left(\sigma, \sigma^{*}\right)=\sum_{n=0}^{\infty} \sum_{m=0}^{\infty} a_{n m} \sigma^{n} \sigma^{* m},
$$

what is the series representation in terms of $x, y$ etc. for $\Phi(z)=A_{k}[F]$. 
We begin by recalling [14]

$$
\frac{n !}{\Gamma(k+n)} m^{n} C_{n}^{k / 2}(\xi)=\mathfrak{Q}_{k}\left[\sigma^{n}\right] \equiv \alpha_{k} \int_{+1}^{-1} \sigma^{n}\left(\zeta-\zeta^{-1}\right)^{k-1} \frac{d \zeta}{\zeta},
$$

where $\xi=\frac{x}{r}, r=|z|$, and $C_{n}^{k / 2}(\xi)$ is a GEgenbauter polynomial [14] It follows then, that

$$
\Phi(z)=\sum_{n=0}^{\infty} \alpha_{n} \frac{n !}{\Gamma(k+n)} r^{n} C_{n}^{k / 2}(\xi)=\mathfrak{Q}_{k}[f], \quad \text { where } f(\sigma)=\stackrel{\infty}{\Sigma} \alpha_{n} \sigma^{n}
$$

for $\mid z:<\epsilon$ sufficiently small.

It is interesting to note that the class of functions $f(\sigma) \varepsilon C^{2}$, where $\sigma=x+\frac{i y}{2}\left(\zeta+\sigma^{-1}\right)$ are not even locally $\left(z \varepsilon N\left(z^{0}\right)\right)$ solutions of the GASPT equation [6]. Consequently, the homogeneous polynomials, $A_{n, v}(z)$ of degree $n$ in $x, y$, which are defined as coefficients ef $\zeta$ in fhe trinomial expansion of $\sigma^{n}$.

$$
\sigma^{n}=\sum_{\nu=-n}^{+n} A_{n, \nu}(z) \zeta^{\nu}
$$

are not in general solution of $(1)$ either $\left({ }^{2}\right)$. It can be shown, nevertheless, that $\Phi(z)=\mathfrak{Q}_{k}[f(\sigma)]$ does generate solutions to (1), and that the set of functions $f_{n}(\sigma) \equiv \sigma^{n}$ generates a complete system of polynomials [14]. To show that $\Phi=\mathfrak{A}_{h}[f(\sigma)], f \varepsilon C^{2}$ is a solution of (1) we need only integrate by parts as before

$$
\begin{gathered}
L^{(k)}[\Phi]=\alpha_{k} \int_{+1}^{-1} L^{(k)}[f(\sigma)]\left(\zeta-\zeta^{-1}\right)^{k-1} \frac{d \zeta}{\zeta} \\
=-\frac{\alpha_{k}}{4} \int_{+1}^{-1} f^{\prime \prime}\left(\zeta-\zeta^{-1}\right)^{k+1} \frac{d \zeta}{\zeta}+\frac{i \alpha_{k} k}{2 y} \int_{+1}^{-1} f^{\prime}\left(\zeta+\zeta^{-1}\right)\left(\tau-\zeta^{-1}\right)^{k-1} \frac{d \zeta}{\zeta}= \\
=-\frac{\alpha_{k}}{4} \int_{+1}^{-1} f^{\prime \prime}\left(\zeta-\zeta^{-1}\right)^{k+1} \frac{d \zeta}{\zeta}+\left.\frac{\alpha_{k} i}{2 y} f^{\prime}(\sigma)\left(\zeta-\zeta^{-1}\right)^{k}\right|_{+1} ^{-1}-\frac{\alpha_{k} i}{2 y} \int_{+1}^{-1} \frac{\partial f(\sigma)}{\partial \zeta}\left(\zeta-\zeta^{-1}\right)^{k-1} \frac{d \zeta}{\zeta}
\end{gathered}
$$

(2) When $y=0$, the polynomial $A_{n y}(\varepsilon)$ is a solution of the GASPT equation for $k=1$, since $A_{n 0}(z)=2 r^{n} P_{n}(\xi)$.

We shall see shortly, that the $A_{n_{v}}(\varepsilon)=$ coustant $r^{n} P_{n}^{\nu}(\xi)$ where the $P_{n}^{\nu}(\xi)$ are associated LEGENDRE functions. 
It may be shown in the same way, that $\Phi=\mathfrak{Q}_{k}\left[f\left(\sigma^{*}\right)\right]$ is also a solution of the GASPT equation.

There is a relation, however, between the polynomials $A_{n, v}(z)$ and associated LEgEndre functions. This may be seen from Heine's integral relation [14],

$$
\begin{aligned}
& \frac{n !}{i^{m}(m+n) !} r^{n} P_{n}^{m}(\xi)=\frac{1}{2 \pi i} \int_{|\zeta|=1}\left(x+\frac{i y}{2}\left(\zeta+\zeta^{-1}\right)\right)^{n} \zeta^{m} \frac{d \zeta}{\zeta}= \\
& =\frac{1}{2 \pi i} \int_{|\zeta|=1} \sigma^{n \zeta} \frac{d \zeta}{\zeta}=A_{n, m}(z)
\end{aligned}
$$

Likewise, the coefficients $A_{n, \mathrm{v}}^{*}(z)$ of the axpansion of $\sigma^{* n}$ also may be related to the LEGENDRE functions by

$$
\begin{gathered}
\frac{i^{m} m !}{(n-m) !} r^{n} P_{n}^{-m}(\xi)=\frac{1}{2 \pi i} \int_{|\zeta|=1}\left(x-\frac{i y}{2}\left(\zeta+\zeta^{-1}\right)\right)^{n} \zeta^{m} \frac{d \zeta}{\zeta}= \\
=\frac{1}{2 \pi i} \int_{|\zeta|=1} \sigma^{*} \zeta^{m} \frac{d \zeta}{\zeta}=A_{n,-m}^{*}(z) .
\end{gathered}
$$

We note in passing, that expressions (13), (14) may be used to give a representation for the GEGENBAUER polynomials in terms of the LEGENDRE functions, when $k>0$.

$$
r^{n} C_{n}^{k_{i} 2}(\zeta)=\frac{4 \Gamma(k+n)}{(4 i)^{k} \Gamma\left(\frac{k}{2}\right)^{2} n !} \int_{\substack{|\zeta|=1 \\|\zeta|}}^{-1} \sigma^{n}\left(\zeta-\zeta^{-1}\right)^{k-1} \frac{d \zeta}{\zeta}
$$

hence

$$
\left.C_{n}^{k / 2}(\zeta)=\frac{4 \Gamma(k+n)}{(4 i)^{k} \Gamma\left(\frac{k}{2}\right)^{2} n !} \stackrel{\Sigma}{m=-n}^{+} i^{m} \frac{P_{n}^{-m}(\xi)}{(n-m) !} \int_{\substack{+1=1 \\|\zeta|=1}}^{-1} \zeta^{m(\zeta}-\zeta^{-1}\right)^{k-1} \frac{d \zeta}{\zeta}
$$

However, it is known $\left({ }^{3}\right)$ that for $k>0$,

$$
\int_{\substack{+1 \\ \xi=1}}^{-1} \zeta^{m}\left(\zeta+\zeta^{-1}\right)^{k-1} \frac{d \zeta}{\zeta}=\frac{\pi i^{k+m}}{k B\left(\frac{k+1+m}{2}, \frac{k+1-m}{2}\right)}, \text { where } B(p, g)=\frac{\Gamma(p) \Gamma(g)}{\Gamma(p+g)}
$$

(3) See page 12 of Higher Trascendental Functions, Vol. I [14]. 
is the Beta-function. One then has

$$
C_{n}^{k / 2}(\xi)=\frac{\pi 4^{1-k}}{k} \frac{\Gamma(k+n)}{\Gamma\left(\frac{k}{2}\right)^{2}} \sum_{m=-n}^{+n} \frac{(-)^{m}}{(n+m) !} \frac{P_{n}^{m}(\xi)}{B\left(\frac{k+1+m}{2}, \frac{k+1-m}{2}\right)}
$$

One may consider the integral

$$
\begin{gathered}
\mathfrak{Q}_{k}\left[\sigma^{n} \sigma^{* m}\right]=\alpha_{k} \int_{\substack{+1 \\
|\zeta|=1}}^{-1} \sigma^{n} \sigma^{* m}\left(\zeta-\zeta^{-1}\right)^{k-1} \frac{d \zeta}{\zeta}= \\
=\alpha_{k} n ! m ! r^{m+n} \sum_{\nu=-n}^{+n} \sum_{\mu=-m}^{+m} i^{\nu-\mu} \frac{P_{n}^{\nu}(\xi) P_{m}^{\mu}(\zeta)}{(n+\nu) !(m+\mu) !} \int_{+1}^{-1} \zeta^{\nu+\mu\left(\zeta-\zeta^{-1}\right)^{k-1}} \frac{d \zeta}{\zeta}= \\
=\frac{\pi}{k} \alpha_{k} i^{k} n ! m ! r^{n+m} \sum_{\nu=-n}^{+n} \sum_{\Sigma=-m}^{+m} \frac{(-)^{\nu}}{(n+\nu) !(m+\mu) !} \frac{P_{n}^{\nu}(\xi) P_{m}^{\mu}(\xi)}{B\left(\frac{k+1+\mu+\nu}{2}, \frac{k+1-\mu-\nu}{2}\right)} .
\end{gathered}
$$

\section{III. - Particular solutions generated by $A_{k}[f]$.}

In order to generate particular solutions of $L^{k}[\Phi]=\rho$ where $\rho$ is analytic about the origin in the real variables $x, y$ we consider the integral equation

$$
\int_{\substack{|+1\\| \zeta \mid=1}}^{-1} g\left(\sigma, \sigma^{*}\right)\left(\zeta-\zeta^{-1}\right)^{k-1} \frac{d \zeta}{\zeta}=\rho(x, y)
$$

where

$$
g\left(\sigma, \sigma^{*}\right)=4 \alpha_{k} \frac{\partial^{2} f}{\partial \sigma \partial \sigma^{*}}
$$

If $\rho(x, y)$ has the TAYLOR espansion.

$$
\rho(x, y)=\sum_{\mu=0}^{\infty} \sum_{\nu=0}^{\infty} a_{\mu \nu} x^{\mu} y^{\nu}
$$


then one may solve formally for the TAYLOR coefficients, $g_{M N}$, of $g\left(\sigma, \sigma^{*}\right)$ as follows.

$$
\begin{aligned}
& a_{m n}=\frac{\partial^{m+n}}{\partial x^{m} \partial y^{n}} \rho(0,0)=\left.\int_{\substack{+1=1 \\
|\zeta|=1}}^{-1} \frac{\partial^{m+n}}{\partial x^{m}} \frac{\partial y^{n}}{g} g\left(\sigma, \sigma^{*}\right)\right|_{x=y=0}\left(\zeta-\zeta^{-1}\right)^{k-1} \frac{d \zeta}{\zeta}= \\
& =\left.\int_{\substack{+1 \\
|\xi|=1}}^{-1}\left(\frac{i}{2}\right)^{n} \sum_{\mu=0}^{m} \sum_{\nu=0}^{n}\left(\begin{array}{l}
m \\
\mu
\end{array}\right)\left(\begin{array}{l}
n \\
\nu
\end{array}\right)(-)^{\nu} \frac{\partial^{m+n} g\left(\sigma, \sigma^{*}\right)}{\partial \sigma^{\mu+\nu} \partial \sigma^{*}(m+n)-(\mu+\nu)}\right|_{\alpha=y-0} \cdot\left(\zeta+\zeta^{-1}\right)^{n}\left(\zeta-\zeta^{-1}\right)^{k-1} \frac{d \zeta}{\zeta}= \\
& =\left(\begin{array}{l}
i \\
2
\end{array}\right) \sum_{\mu=0}^{m} \sum_{\nu=0}^{n}\left(\begin{array}{l}
m \\
\mu
\end{array}\right)\left(\begin{array}{l}
n \\
\nu
\end{array}\right)(-)^{v} g_{\mu+\nu, m+n-(\mu+\nu)} \int_{\substack{+1 \\
|\zeta|=1}}^{-1}\left(\zeta+\zeta^{-1}\right)^{n}\left(\zeta-\zeta^{-1}\right)^{k-1} \frac{d \zeta}{\zeta},
\end{aligned}
$$

or

$$
a_{m n}=\beta_{n k} \sum_{\mu=0}^{m} \sum_{\nu=0}^{n}(-)^{\nu}\left(\begin{array}{l}
m \\
\mu
\end{array}\right)\left(\begin{array}{l}
n \\
\nu
\end{array}\right) g_{\mu+\nu,(m+n)-(\mu+\nu)},
$$

where

$$
\beta_{n k}=\left(-\frac{1}{2}\right)^{n} k \pi i^{k} \sum_{\nu=0}^{n} \frac{(-)^{\nu}\left(\frac{n}{\nu}\right)}{B\left(\frac{k+1-n}{2}-\nu, \frac{k+1+n}{2}-\nu\right)} .
$$

Several special cases are suggested imediately, for instance, where $\rho$ is a function of $x$ or $y$ alone. If $p(x, y) \equiv p(x)$, we may then choose $g\left(\sigma, \sigma^{*}\right)$ to have the form $G\left(\sigma+\sigma^{*}\right) \equiv G(2 x)$; one then has

$$
g\left(\sigma, \sigma^{*}\right)=G(2 x)=\frac{p(x)}{\beta_{0} k}, \quad \beta_{0 k}=\frac{i^{k} \pi}{k B\left(\frac{k+1}{2}, \frac{k+1}{2}\right)},
$$

and

$$
f\left(\sigma, \sigma^{*}\right)=\frac{p(x)}{4 \alpha_{k} \beta_{0 k}} \sigma \sigma^{*}
$$

A particular solution to the Porsson equation is then given by

$$
\begin{aligned}
& \Phi(z)=\frac{p(x)}{4 \beta_{0 k}} \int_{\substack{+1 \\
|\bar{\xi}|=1}}^{-1} \sigma \sigma^{*}\left(\zeta-\zeta^{-1}\right)^{k-1} \frac{d \zeta}{\zeta}= \\
& =\pi \frac{\pi}{k} r^{2} i^{k} p(x) \sum_{\nu=-1}^{+1} \sum_{\mu=-1}^{+1} \frac{(-)^{\nu}}{(1+\nu) !(1+\mu) !} \frac{P_{1}^{\nu}(\zeta) P_{1}^{\nu}(\zeta)}{B\left(\frac{k+1+\mu+\nu}{2}, \frac{k+1-\mu-\gamma}{2}\right)} .
\end{aligned}
$$


In the case $\rho(x, y) \equiv p(y), g\left(\sigma, \sigma^{*}\right)$ may be represented as $g=G\left(\sigma-\sigma^{*}\right)=$ $=G\left(i y\left[\zeta+\zeta^{-1}\right]\right)$, and we consider then the integral equation,

$$
\int_{|+|=1}^{-1} G\left(i y\left[\zeta+\zeta^{-1}\right]\right)\left(\zeta+\zeta^{-1}\right)^{k-1} \frac{d \zeta}{\zeta}=p(y)
$$

If $G(z)=\sum_{\nu=0}^{\infty} g_{\nu} z^{\nu}$, and $p(y)=\sum_{\nu=0}^{\infty} p_{\nu} y^{\nu}$ one may compute the TAYLOR coefficients $g_{y}$ formally as follows.

$$
\begin{gathered}
\int_{\substack{\begin{subarray}{c}{1 \\
|\zeta|=1} }} \\
{\sum_{n=0}^{\infty} g_{n}}\end{subarray}}^{\sum_{\nu=0}^{n}(i y)^{n}\left(\begin{array}{l}
n \\
\nu
\end{array}\right) \zeta^{2 \nu-n}\left(\zeta-\zeta^{-1}\right)^{k-1} \frac{d \zeta}{\zeta}=\sum_{n=0}^{\infty}(i y)^{n} g_{n} \sum_{\nu=0}^{n}\left(\begin{array}{l}
n \\
\nu
\end{array}\right) \int_{\substack{+1 \\
|\zeta|=1}}^{-1} \zeta^{2, \nu-n}\left(\zeta-\zeta^{-1}\right)^{k-1} \frac{d \zeta}{\zeta}=} \\
=\pi k i^{k} \sum_{n=0}^{\infty} g_{n}(-y) \sum_{\nu=0}^{n}\left(\begin{array}{l}
n \\
\nu
\end{array}\right)(-)^{\nu} / B\left(\frac{k+1-n}{2}+\nu, \frac{k+1+n}{2}-\nu\right) ;
\end{gathered}
$$

from which we have

$$
g_{n}=\frac{(-)^{n} p_{n}}{\pi k i_{k} \gamma_{k n}}, \quad \text { where } \gamma_{k n} \equiv \sum_{\nu=0}^{n}\left(\begin{array}{l}
n \\
\nu
\end{array}\right)(--)^{\nu} / B\left(\frac{k+1-n}{2}+\nu, \frac{k+1+n}{2}-\nu\right)
$$

providing $\left|\gamma_{k n}\right| \geq a>0$, for all $n$, and for $k>0$. One may preceed as before and oblain a particular solution,

$$
\left.\left.\Phi=\int_{\substack{+1 \\|\zeta|=1}}^{-1} \sigma \sigma^{*} G\left(i y \mid \zeta+\zeta^{-1}\right]\right) \zeta-\zeta^{-1}\right)^{k-1} \frac{d \zeta}{\zeta} .
$$

\section{IV. - The Poisson Equation in Three Variables.}

It is interesting to note that the methods developed in the previous section may be extended to PoIsson's equation in three variables, that is we consider the equation

$$
\triangle u \equiv \frac{\partial^{2} u}{\partial x_{1}^{2}}+\frac{\partial^{2} u}{\partial x_{2}^{2}}+\frac{\partial^{2} u}{\partial x_{3}^{2}}=\rho\left(x_{1}, x_{2}, x_{3}\right)
$$


We may generate solutions to this equation by means of an integral representation similar to the WHITTAKER-BERGMAX operator which maps functions of two complex variables into solutions of LAPLACE's equations, $\Delta H(x)=0$ [11], [12], [13].

$$
H(X)=p_{3}(f) \equiv \frac{1}{2 \pi i} \int_{2} f(t, \zeta) \frac{d \zeta}{\zeta},
$$

$$
t=\left[-\left(x_{1}-i x_{2}\right) \frac{\zeta}{2}+x_{3}+\left(x_{1}+i x_{2}\right) \frac{1}{2 \zeta}\right], \quad\left\|X-X^{0}\right\|<\epsilon, \quad X \equiv\left(x_{1}, x_{2}, x_{3}\right)
$$

$X^{0} \equiv\left(x_{1}^{0}, x_{2}^{0}, x_{3}^{0}\right)$, where $\mathcal{L}$ is a closed differentiable are in $\zeta$-plane, and $\epsilon>0$ is sufficiently small.

Following our previous method we introduce the integral representation $\Phi_{3}(F)$,

$$
u(X)=\Phi_{3}\left(F^{\prime}\right) \equiv \frac{1}{2 \pi i} \int_{\mathcal{L}} F\left(t, t^{*}, \zeta\right) \frac{d \zeta}{\zeta},
$$

where $F$ is an analytic function of the three complex variables $t, t^{*}, \zeta ; t$ is the same as above,

$$
t^{*}=\left[\left(x_{1}-i x_{2}\right) \frac{\zeta}{2}+x_{3}-\left(x_{1}+i x_{2}\right) \frac{1}{2 \zeta}\right], \quad\left\|X-X^{0}\right\|<\epsilon, \text { etc.. }
$$

It is clear, that for $|\zeta|=1$ and $X \equiv\left(x_{1}, x_{2}, x_{3}\right)$ a real point, then $t^{*}=\bar{t}$. If $\frac{\partial F}{\partial t}=0$, or $\frac{\partial F}{\partial t^{*}}=0$, then $F$ is a function of $t$ or $t^{*}$ respectively; in these cases $\Phi(F)$ becomes the WhImTakgR-Berguan operator, and $u(X)$ is a harmonic function. If $\rho(X) \varepsilon C^{2}$, then we-have by interchanging orders of differentiation and integration

$$
\rho(X)=\Delta u=\frac{1}{2 \pi i} \int_{|\zeta|=1} \Delta F \frac{d \zeta}{\zeta}=\frac{1}{4 \pi i} \int_{|\zeta|=1}\left(\zeta-\zeta^{-1}\right)^{2} \frac{\partial^{2} F}{\partial t \partial t^{*}} \frac{d \zeta}{\zeta} .
$$

Hence, as before we may obtain a particular solution of $\Delta u=\rho$, with the representation (22) if $F$ is a solution of the integral equation (23). In order, to see how $\Phi(F)$ transforms analytic functions of three variables into solutions 
of (20), we first consider the integrals

$$
\begin{aligned}
& u_{n, m, \lambda}(X)=\Phi\left(t^{n} t^{* m} \zeta^{\lambda}\right) \equiv \frac{1}{2 \pi i} \int_{|\zeta|=1} t^{n} t^{* m} \zeta^{\lambda} \frac{d \zeta}{\zeta}, \quad-n-m \leq \lambda \leq n+m, \\
& =\frac{1}{2 \pi i} r^{n+m} n ! m ! \int_{|\zeta|=1}\left(\sum_{\nu=-n}^{+n} P_{n}^{\nu}(\xi) \frac{i^{-\nu \zeta^{-\nu}}}{(n+\nu) !} e^{i \nu \varphi}\right)\left({\underset{\Sigma}{\Sigma}}_{\Sigma=-m}^{+m}(-)^{\mu} \cdot P_{m}^{\mu}(\xi) \frac{i^{-\mu \zeta-\mu}}{(m+\mu) !} e^{i \mu \varphi}\right) \zeta^{\lambda} \frac{d \zeta}{\zeta}=
\end{aligned}
$$

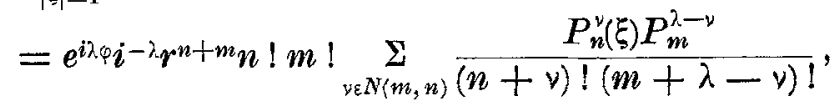

where $N(m, n)$ is the set of indices $\{\nu|\nu| \leq n,|\lambda-\nu| \leq m \mid$. Consequently, if $F$ is the analytic function

$$
F\left(t, t^{*}, \zeta\right)=\sum_{n, m=0}^{\infty} \sum_{\lambda=-n-m}^{n+m} a_{+m \lambda} t^{n} t^{* m} \eta^{\lambda},
$$

defined in the region $|t|<R,\left|t^{*}\right|<R, 1-t<|\zeta|<1+t$, the corresponding function $u(X)$, which is regular in a neighborhood of the origin is

$$
u(X)=\sum_{n, m=0}^{\infty} \sum_{\lambda=-n-m}^{n+m} a_{n i m \lambda} u_{n m \lambda}(X)
$$

Since, the integral (24) vanishes whenever $|\lambda|>m+n$ we need just consider the functions $F$ defined by (25), we shall realize that this class of functions does not in general generate solutions to $\triangle u=p$, when $p$ is an arbitrary real analytic function of $x_{1}, x_{2}, x_{3}$.

Under certain conditions the integral equation (23) may be solved; to see when this is possible we consider the integral

$$
\frac{1}{\alpha \pi i} \int_{|\zeta|=1} G\left(t, t^{*}, \zeta\right)\left(\zeta-\zeta^{-1}\right)^{2} \frac{d \zeta}{\zeta}, \quad \text { where } G=\frac{1}{2} \frac{\partial^{2} F}{\partial t \partial t^{*}}
$$

and $G$ has a representation such as (25). The general term in the series expansion for $G$ integrates as follows

$$
\begin{gathered}
v^{n m \lambda}(X)=\frac{1}{\alpha \pi i} \int_{|\zeta|=1} t^{n} t^{* m} \zeta^{\lambda}\left(\zeta-\zeta^{-1}\right)^{2} \frac{d \zeta}{\zeta}= \\
=n ! m ! r^{n+m} \sum_{\nu=-n}^{+n} \sum_{\nu=-m}^{+m} \frac{P_{\nu}^{n}(\xi) P_{n}^{m}(\xi) e^{i(\nu+\mu) \varphi}}{i^{\mu+\nu}(n+\nu) !(m+\mu) !} \frac{1}{\alpha \pi i} \int_{|\zeta|=1} \zeta^{\lambda-\mu-\nu\left(\zeta-\zeta^{-1}\right)^{2}} \frac{d \zeta}{\zeta}= \\
=-i^{\lambda} n ! m ! r^{n+m} \sum_{\nu=-n}^{+n} \sum_{\mu=-m}^{+m}(-)^{\mu+\nu} \frac{P_{\nu}^{n}(\xi) P_{\mu}^{m}(\zeta) e^{i(\nu+\mu) \varphi}\left[1+e^{i(\lambda-\nu-\mu) \pi}\right]}{(n+\nu) !(m+\mu) ! \Gamma\left(2+\frac{\lambda-\mu-\nu}{2}\right) \mathrm{T}\left(2-\frac{\lambda-\mu-\nu}{2}\right)} .
\end{gathered}
$$


If $\rho\left(x_{1}, x_{2}, x_{3}\right)$ may be expanded in terms of the polynomials $\left\{\gamma_{n m \lambda}\right\}$, we may find a function $G$ which satisfies (23), and consequently generate all solutions of (20). This clearly is not the case in general; however, under certain criteria of symmetry on $\rho\left(x_{1}, x_{2}, x_{3}\right)$ such an espansion will be possible.

\section{REFERENCES}

[1] Weinstein, Discontinuous integrals and generalized potential theory "Trans. Amer. Math. Soc. * Vol. 63 (1948) pp. 342.454.

[2] - - Generalized axially symmetric potential theori, «Bull. Amer. Math. Soc. \& Vol. 59 (1958) pp. $20-38$.

[3] - - The method of axial symmetry in partial differential equations; Edizioni Cremo* nese (Roma 1955) pp. 1.10.

[4] - - On a class of partial differential equations of even order, "Annali di matematica pura ed applicatan, Serie IV. Tomo XXXIX (1956), pp. 245-25t.

[5] R. GILBERT, On the singularities of generalned axially symmetric potentials "Areh. Rat. Mech. Anal. Vol. 6, No 2, (1960) pp. 171:176.

[6] - -, On generalized axially symmetric potentials (to appear in die J. Reine u. Angew, Math.).

[7] - - Some properties of generalized axially symmetric potentials Amer. J. Math. LXXXIV, No. 3, (1962).

[8] - -, Composition formulae in generalized axially symmetric potential theory (to appear).

[9] P. HenRIor, On the domain of regularity of generalized axially symmetric potentials, - Proc. Math. Soc, Vol. 8, (1957) pp. 29.31.

[10] A. G. MACKJE, Contour integral solutions of a class of differential equations, . R. Rat. Mech. Anal. ", Vol. 4, (1955) pp. 733750.

[11] S. BERGMAN, Zur Theorie der algebraischen potential funktionen des drei dimensionalen Raumes, "Math. Ann. Vol. 99, (1928) pp. 629.650, and Vol. 101 (1929) pp. 534-548.

[12] - -, Residue theorems of harmonic functions of three variables, «Bull. Amer. Math. Soc. ", Vol ${ }_{r}$ 49, No 2. pp. 163.174 (1943).

[13] - -, Some properties of a harmonic function of three variables given by its series development, "Arch. Rat.: Mech Anal. *, Vol. 8, No 3, (1962), pp. 211.226.

[14] A.' ERDÉLXI et al, Higher Transcendental Functions, Vol. I and II (New York), 1953.

[15] - - Singularities of Generalized axially symmetric potentials, «Comm. Pure Appl. Math. » Vol. 9, (1956), pp. 408-414. 